\title{
Memory and Learning Improvement by Resveratrol and Probiotics via the Gut-brain Axis and Antioxidant Activity in Diabetic Rats
}

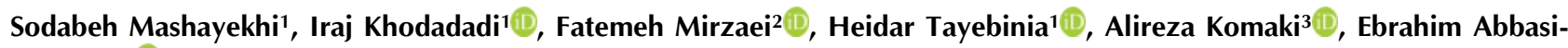 \\ Oshaghi ${ }^{*}$ (D)
}

1 Department of Clinical Biochemistry, Hamadan University of Medical Sciences, Hamadan, Iran

${ }^{2}$ Department of Anatomy, School of Medicine, Hamadan University of Medical Sciences, Hamadan, Iran

${ }^{3}$ Neurophysiology Research Centre, Hamadan University of Medical Sciences, Hamadan, Iran

\section{*Corresponding author:}

Ebrahim Abbasi Oshaghi, Department

of Clinical Biochemistry, Hamadan

University of Medical Sciences,

Hamadan, Iran

Tel: +988138380208

Email: a.oshaghi@umsha.ac.ir

7abbasi@gmail.com
Received: 20 Aug. 2020 Accepted: 11 Sep. 2020 ePublished: 01 May. 2021

\begin{abstract}
Background and Objective: Diabetes affects the central nervous system associated with cognition, especially memory and learning. The present study aimed to investigate the effects of probiotics (living microorganisms that provide health benefits) and resveratrol (a polyphenol with potential antioxidant activity) combination on oxidative stress, glucagon-like peptide-1 (GLP-1), memory, and learning in diabetic rats.

Materials and Methods: Male Wistar rats were randomly divided into five groups (six animals per group) of control, diabetic, probiotic-treated diabetic $\left(50410^{9} \mathrm{CFU} / \mathrm{kg}\right.$ in drinking water), resveratroltreated diabetic (10 mg/kg, oral gavage), as well as probiotics and resveratrol-treated diabetic. The treatment procedures lasted for four weeks, and a Shuttle Box test was then performed to evaluate memory and learning. At the end of the study, animals were sacrificed, and the hippocampus was removed to perform biochemical studies.

Results: The levels of malondialdehyde and total oxidative status significantly decreased in the diabetic group treated with combined resveratrol and probiotics $(\mathrm{P}<0.05)$. Furthermore, the levels of superoxide dismutase, catalase, and glutathione peroxidase significantly increased in the hippocampus of the diabetic group treated with combined resveratrol and probiotics $(\mathrm{P}<0.05)$ According to the results, the combined therapy improved memory and learning $(\mathrm{P}<0.05)$. In addition, the level of GLP-1 increased in the treatment groups $(\mathrm{P}<0.05)$.

Conclusions: Treatment with resveratrol and probiotics significantly normalized pyramidal cell densities in the hippocampus of diabetic rats. This combination also reduced oxidative stress and activated the gut-brain axis in diabetic animals.

Keywords: Diabetes, Glucagon-like peptide-1, Memory and learning, Oxidative stress probiotics, Resveratrol
\end{abstract}

\section{Background}

Diabetes is a chronic metabolic disease and is characterized by insulin resistance and loss of pancreatic $\beta$-cell function $[1,2]$. Hyperglycemia is one of the central factors for the development of oxidative stress that induces important complications in diabetic patients [3]. These complications typically include microvascular (retinopathy, neuropathy, and nephropathy) and macrovascular (cardiovascular and cerebrovascular) diseases [4].

Diabetes affects the central nervous system (CNS) associated with cognition, especially memory and learning; moreover, it increases the risk of developing dementia by up to $60 \%$ [5]. Hyperglycemia enhances the formation of reactive oxygen species (ROS) through glucose autoxidation, polyol pathways, and non-enzymatic glycation of proteins. These free radicals can have significant effects on carbohydrates, proteins, and DNA [6].
The hippocampus is a part of the limbic system of the brain, and it is the consolidation center for cognitive functions, such as memory and learning that are affected in diabetes [7]. The gut-brain axis (GBA) has been introduced as a novel therapeutic target in neurological disorders and other diseases that function through glucagon-like peptide-1(GLP1) [8]. Additionally, GLP-1 and its analogs can cross the blood-brain barrier and improve memory and learning [9].

Studies have indicated that probiotics can suppress the production of oxidative stress by increasing the levels of antioxidant enzymes, such as superoxide dismutase (SOD) and glutathione peroxidase (GPx) [10,11]. Previous studies also revealed that probiotics were beneficial for brain function and had therapeutic effects for various CNS disorders [12]. Another way to treat metabolic disorders is the 
use of natural compounds rich in phenols and flavonoids [13-16]. Resveratrol is a polyphenol that can cross the blood-brain barrier in the areas of the brain that are involved in learning and memory and may be involved in modulating message transmission in CNS [17]. Consequently, it has been suggested as a potential compound in the treatment of diabetes and neurodegenerative disorders [18]. Probably, the concurrent administration of these compounds can have potent neuroprotective effects.

\section{Objectives}

The present study aimed to investigate the therapeutic effects of probiotics and resveratrol combination on oxidative stress, memory, learning, as well as GLP-1 levels in Streptozotocin (STZ) induced diabetic rats.

\section{Materials and Methods \\ Animals}

This experimental study was conducted on 30 healthy 8-week-old Wistar rats (weight range: 250300) that were purchased from Hamadan University of Medical Sciences, Hamadan, Iran. The rats were kept under a conditioner at 50\%-60\% humidity and a controlled temperature of $22 \pm 2^{\circ} \mathrm{C}$ within the animal room under a 12:12-h light/dark cycle. Subsequently, the rats were randomly divided into five groups of six animals per group and kept in separate cages. The experimental groups included: 1) control rats, 2) diabetic rats, 3) diabetic rats receiving probiotic supplements $\left(50 \times 10^{\circ} \mathrm{CFU} / \mathrm{kg}\right)$, 4) diabetic rats receiving resveratrol $(10 \mathrm{mg} / \mathrm{kg})$, and 5) diabetic rats receiving the combined probiotic and resveratrol supplements.

\section{Induction of Type 2 diabetes}

To induce type 2 diabetes, single doses of STZ (65 $\mathrm{mg} / \mathrm{kg}$ ) (Sigma-Aldrich, USA) and nicotinamide $(110 \mathrm{mg} / \mathrm{kg})$ were administered intraperitoneally (dissolved in $0.1 \mathrm{M}$ citrate buffer, $\mathrm{pH}=4.5$ ) [3]. Furthermore, blood glucose levels were measured by glucometer seven days after STZ injection and regarded as type 2 diabetes if their blood glucose was above $250 \mathrm{mg} / \mathrm{dl}$.

\section{Animal treatments}

Probiotics contain lyophilized bacteria, including eight different strains (Bifidobacterium longum, Bifidobacterium breve, Bifidobacterium infantis, Lactobacillus acidopbilus, Lactobacillus plantarum, Lactobacillus casei, and Lactobacillus bulgaricus) with a dose of $50 \times 109 \mathrm{CFU} / \mathrm{kg}$ in drinking water for four weeks according to previously published studies [3]. Totally, $10 \mathrm{mg} / \mathrm{kg}$ of resveratrol powder was daily mixed in $1 \mathrm{ml}$ sterile distilled water per rat and maintained on ice until complete gavage [19]. The animals received probiotics and resveratrol once a day for four weeks. It should be noted that the behavioral tests were performed at the end of the treatment. Furthermore, the probiotics and resveratrol were purchased from Genuine Health (Canada) and Natural Fact Companies (USA), respectively.

\section{Behavioral tests}

Evaluation of passive avoidance learning

The passive avoidance learning (PAL) test was performed at the end of the experiment using a Shuttle Box. This plexiglass box is divided into both dark and light compartments with a dimension of $30 \times 20 \times 30 \mathrm{~cm}$. The two compartments are interconnected by a single $(20 \times 50 \mathrm{~cm})$ valve connected by a guillotine door. In total, three $\mathrm{mm}$ metal bars with a distance of $10 \mathrm{~mm}$ were installed on the floor of the plexiglass box. A dark chamber was considered during testing, and the guillotine door was closed between the two compartments during the training. When the rat was placed in the light compartment, the guillotine door was opened, and the delay time was measured by a chronometer. After the entrance of the rat to the dark compartment, the guillotine door was closed, and an electric shock was applied to the animals during the training phase through bars attached to the stimulator device. At this stage, the rats were placed in the light part to be acquainted with the device, and after $30 \mathrm{sec}$, the door was opened between the two parts. After the rat entered the dark area, the door was closed and after $30 \mathrm{sec}$, the animal was taken out and returned to the cage. Subsequently, these steps were repeated after $30 \mathrm{~min}$, and following the second stage, the rat was placed in a bright environment for $30 \mathrm{sec}$, and then the guillotine door was opened between the two parts. The delayed entry time (step-through latency) (STLa) was recorded for each rat at that stage. After the rat had fully entered the dark chamber, the guillotine door was closed, and an electric shock was applied through the metal rods of the chamber floor for a second. The animal was then removed from the dark chamber after $30 \mathrm{sec}$ and returned to the cage. This step was repeated after $2 \mathrm{~min}$, and the retention test was performed according to a previously conducted study [20]. The rats were placed in a light chamber (the same as PAL training), and $5 \mathrm{sec}$ later, the guillotine door was opened, and the STLa in the retention trial (STLr), as well as the time spent in dark compartment (TDC), were recorded for up to $300 \mathrm{sec}$. If the rat did not enter the dark chamber within $300 \mathrm{sec}$, the retention test was terminated and a ceiling score of 
$300 \mathrm{~s}$ was assigned.

Tissue measurement of GLP-1

The ELISA Kit was used to determine the GLP-1 levels according to the manufacturer's instructions (ZellBio, Germany).

Determination of total antioxidant capacity, total oxidative status, and malondialdehyde

Total antioxidant capacity (TAC) was determined by ferric-reducing antioxidant power assay [21,22]. Furthermore, the total oxidative status (TOS) of the sample was determined by the oxidation of ferrous iron to ferric in the samples with moderate acidity [21,22]. Malondialdehyde (MDA) level was measured according to the manufacturer's instructions (ZellBio, Germany) [21,22].

Measurement of antioxidant enzyme activity

The activity of catalase (CAT), SOD, and GPX enzymes was measured using a kit (ZellBio, Germany).

Histology of hippocampus

Hippocampus were fixed in $4 \%$ formaldehyde, immersed in paraffin, and then stained with hematoxylin and eosin according to previously conducted studies $[23,24]$.

\section{Statistical analysis}

The results were analyzed using SPSS software (version 20) through ANOVA and Tukey's post hoc test in case of significant results. A p-value less than $0.05(\mathrm{P}<0.05)$ was considered statistically significant.

\section{Results}

Findings of passive avoidance memory test using the Shuttle Box test

The result showed no significant difference among the animals regarding the latency to enter the dark box during the adaptation phase (Figure 1).

Comparison of the time of "first dark entry" in the step-through latency in the retention test

A significant decrease was observed in the time of "first dark entry" in the avoidance memory test in the diabetic group, compared to the control group $(\mathrm{P}<0.001)$. Moreover, the time of "first dark entry" in the probiotics alone and resveratrol alone groups indicated a significant increase $(\mathrm{P}<0.01)$. Furthermore, a comparison of the diabetic with the diabetic group treated with probiotics and resveratrol indicated a significant decrease in this regard $(\mathrm{P}<0.001)$ (Figure 1).

Comparison of the duration of stay in the dark room of the Shuttle Box

After comparing the duration of stay in the dark room of the Shuttle Box in the avoidance memory test, the results indicated a significant decrease in the control group, compared to the diabetic group $(\mathrm{P}<0.001)$. Furthermore, the duration of stay in the dark room of the Shuttle Box in probiotics alone and resveratrol alone groups indicated a significant decrease in this regard $(\mathrm{P}<0.01)$. In addition, the combined probiotics and resveratrol group, compared to the diabetic group, showed a significant decrease in dark room stay $(\mathrm{P}<0.001)$ (Figure 1).

Effect of treatment with probiotics and resveratrol on malondialdehyde concentration

The level of MDA was significantly higher in the diabetic group, compared to the control group $(\mathrm{P}=0.0034)$. Moreover, the level of MDA was significantly decreased in the probiotic-treated diabetic group, compared to the diabetic group (39\% decrease) $(\mathrm{P}=0.093)$. However, there was no
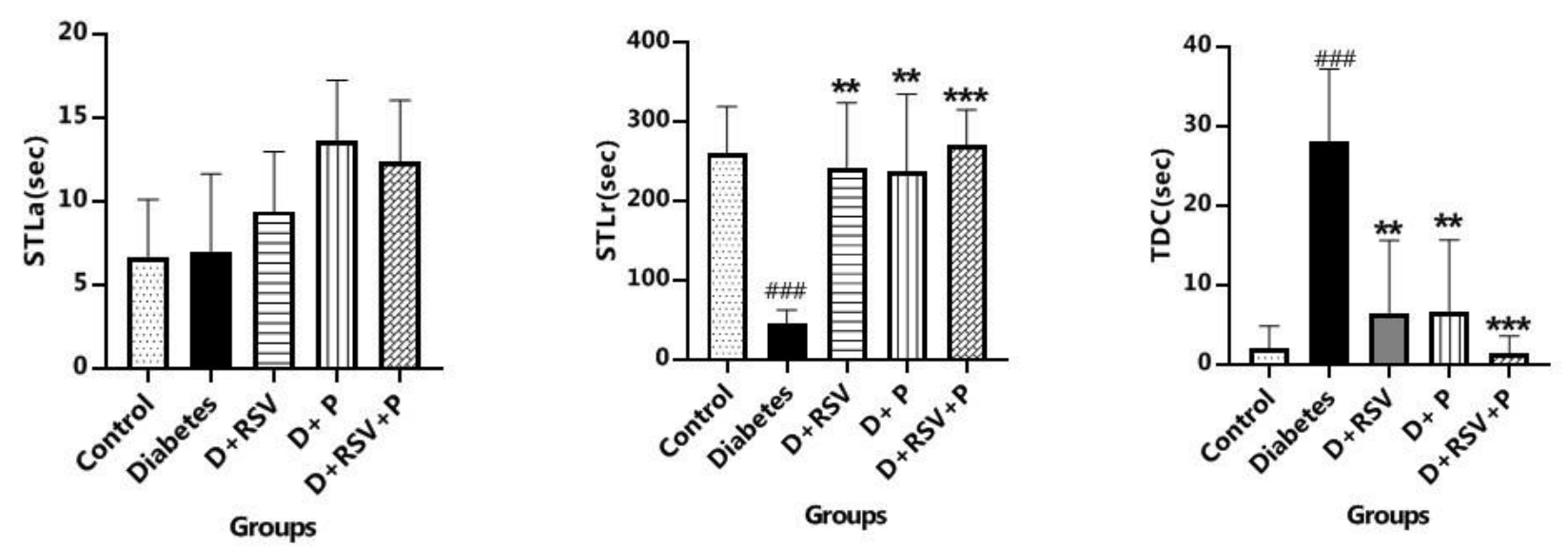

Figure 1. Average latency time to enter the dark compartment (A), time of "first dark entry" in the avoidance memory test (B), and duration of stay in the dark room of the Shuttle Box (C).

Data are presented as means \pm SEM. ${ }^{* *} \mathrm{P}<0.01$ and ${ }^{* * *} \mathrm{P}<0.001$ were set as significant, compared to diabetes; $\# \# \mathrm{P}<0.001$ compared to controls. D: diabetes, RSV: resveratrol, P: probiotic, STLr: step-through latency, RSV: resveratrol, P: probiotic. 

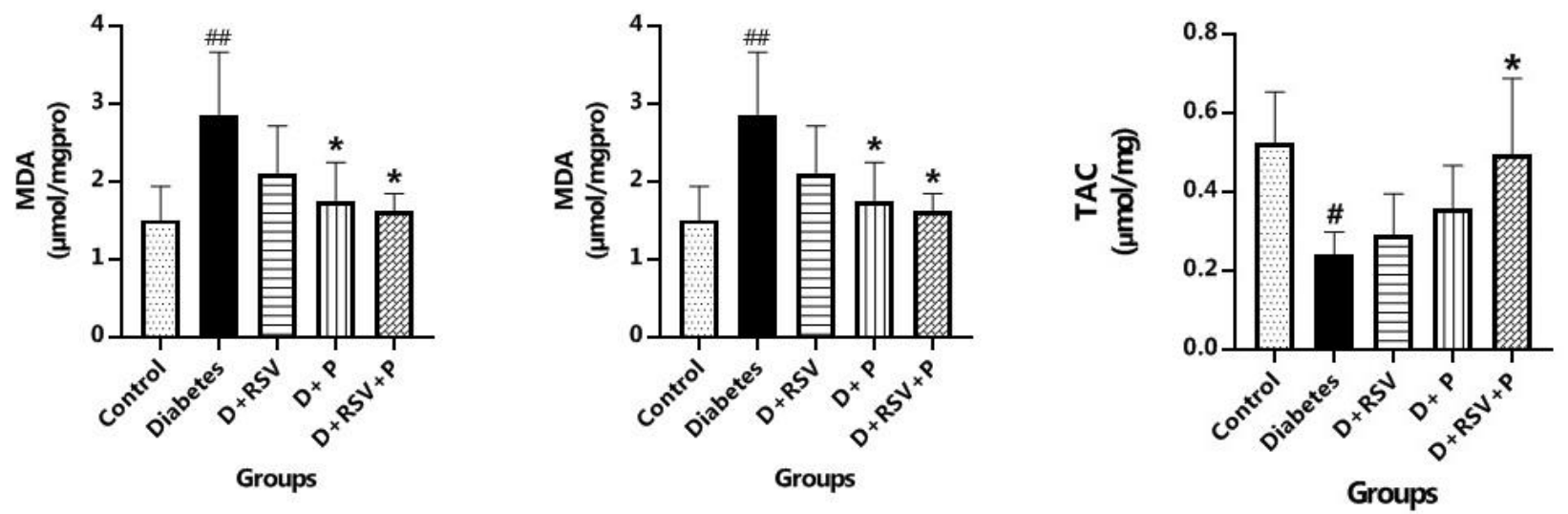

Figure 2. Malondialdehyde, total oxidative status, and total antioxidant capacity levels in different treated groups.

Data are presented as means \pm SEM. ${ }^{*} \mathrm{P}<0.05$ and ${ }^{* *} \mathrm{P}<0.01$ were set as significant, compared to diabetes; \#\# $<0.01$, compared to controls. D: diabetes, RSV: resveratrol, P: probiotic, MDA: Malondialdehyde, TOS: total oxidative status, TAC: total antioxidant capacity.

significant decrease in the resveratrol-treated diabetic group, compared to the diabetic group. On the other hand, there was a significant decrease in the diabetic group treated with resveratrol and probiotics, compared to the diabetic group (44\% decrease) $(\mathrm{P}=0.0185)$ (Figure 2).

\section{Effect of treatment with probiotics and resveratrol} on total oxidative status

The level of TOS as a marker of oxidant showed a remarkable increase in the diabetic group, compared to the control group $(\mathrm{P}=0.0036)$. The level of TOS was significantly decreased in the resveratrol-treated diabetic group, compared to the diabetic group (45\% decrease; $\mathrm{P}=0.0062$ ). Combined probiotics and resveratrol treatment reduced the level of TOS significantly $(52 \%$ decrease; $\mathrm{P}=0.0015)$, which indicated the effect of oxidant decrease in response to combined therapies (Figure 2).

Effect of treatment with probiotics and resveratrol on total antioxidant capacity

The level of TAC was significantly $(\mathrm{P}=0.0192)$ lower in the diabetic group, compared to the control group. Moreover, the increase in the level of TAC was not significant in the diabetic group receiving resveratrol alone and probiotics alone; however, the combined probiotics and resveratrol treatment increased TAC significantly (105\% increase; $\mathrm{P}=0.04$ ) (Figure 2).

Effect of treatment with probiotics and resveratrol on superoxide dismutase, activity of catalase, and glutathione peroxidase activity

The level of SOD activity significantly reduced in response to the combined probiotics and resveratrol treatment $(47 \%$ decrease; $\mathrm{P}<0.001)$. The level of enzyme activity in the combined probiotics and resveratrol treatment group was similar to that of the control group, indicating that this combination therapy was effective (Figure 3). The decrease in CAT enzyme activity was significant in the combined treatment group ( $45 \%$ decrease; $\mathrm{P}=0.01)$. Furthermore, the level of enzyme activity in the combined probiotics and resveratrol treatment group was similar to that of the control group, indicating the positive effect of the combined treatment (Figure 3). Furthermore, the combined
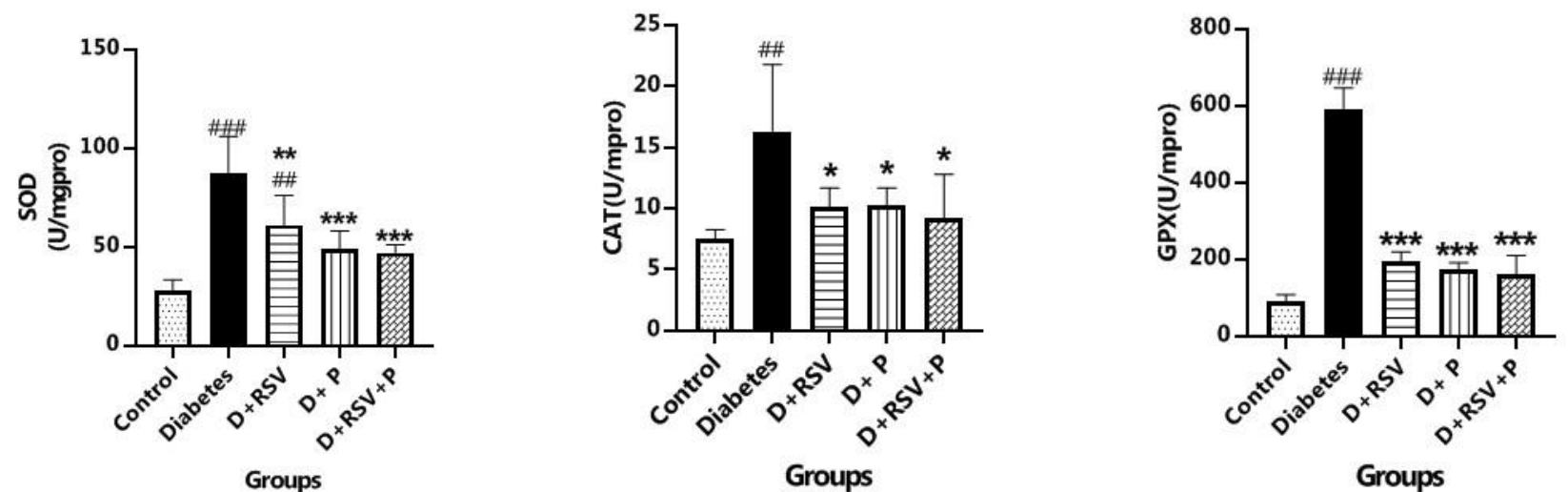

Figure 3. Dismutase, catalase, and glutathione peroxidase levels in different treated groups.

Data are presented as means \pm SEM. ${ }^{* * P}<0.01$ and ${ }^{* * * P}<0.001$ were set as significant, compared to diabetes; \#\# $\mathrm{P}<0.01$ and $\# \# \#<0.001$, compared to controls. D: diabetes, RSV: resveratrol, P: probiotic, SOD: dismutase, CAT: catalase, GPX: glutathione peroxidase. 
treatment was also able to significantly decrease the activity of the GPX enzyme ( $<<0.001)$. The level of enzyme activity in the treatment groups was similar to that of the control group (Figure 3).

Effect of treatment with probiotics and resveratrol on glucagon-like peptide-1

The concentration level of GLP-1 in diabetic rats was significantly lower than that in the control group $(\mathrm{P}<0.001)$. Moreover, the level of GLP-1 significantly increased in the probiotics and resveratrol group, compared to the diabetic group $(\mathrm{P}<0.01)$. The combination of probiotics and resveratrol significantly increased the level of GLP1(Figure 4).

Histology of hippocampus

Hematoxylin and eosin-stained neuron densities showed that the diabetic rats showed a significant decrease in pyramidal cell densities, compared to the control group. However, treatment resveratrol and probiotics significantly normalized these changes (Figure 5).

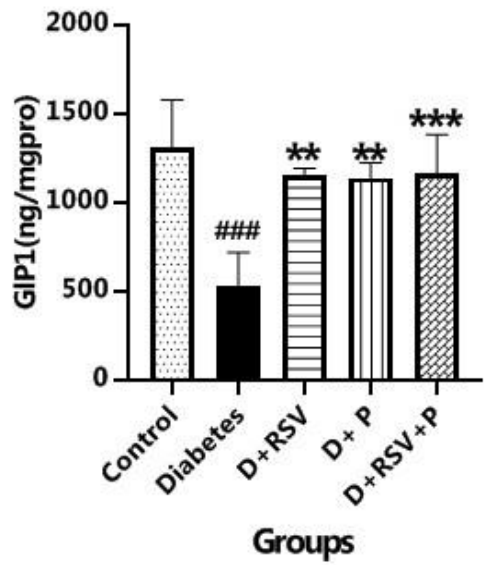

Figure 4. Glucagon-like peptide-1 levels in different treated groups.

Data are presented as means \pm SEM. ${ }^{* * P}<0.01$ and ${ }^{* * *} \mathrm{P}<0.001$ were set as significant, compared to diabetes; $\# \# P<0.001$ compared to controls. D: diabetes, RSV: resveratrol, P: probiotic, GLP-1: glucagon-like peptide-1.
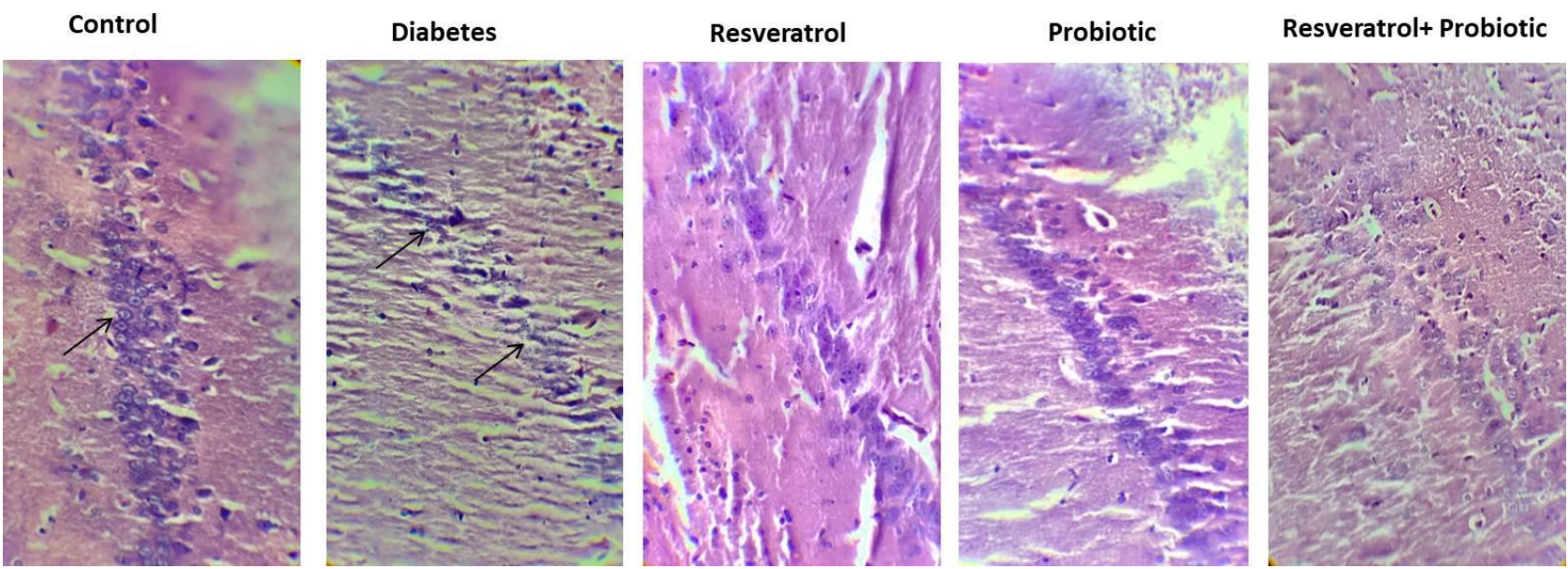

Figure 5. Histological changes of hippocampus determined by hematoxylin and eosin (magnification 4004). Diabetic animals showing reduced thickness of pyramidal cell layer in the CA1 with elevated apoptotic neurons irregular with chromatolysis. Treatment with resveratrol and probiotics normalizing these changes.

\section{Discussion}

Current medications used for the treatment of brain damage may have side effects. Therefore, there is an increasing demand for natural compounds with anti-hyperglycemic properties and fewer side effects [25]. The levels of GLP-1 were decreased in the brain of diabetic rats in the present study. Furthermore, the combination and alone treatment, compared to the diabetic group, increased the GLP1 level. Isik et al. have shown that GLP-1 can protect neurons from oxidative stress damage and has a role in enhancing neurogenesis, as well as reducing apoptosis and inflammation [26]. According to this result, probiotics and resveratrol, as antioxidant compounds, release GLP-1 and probably bind to its receptors in the brain; consequently, they reduce the free radicals and increase neuronal regeneration that in turn can increase memory and learning in diabetic rats.

The results of the present study revealed a decrease in TAC and an increase in TOS in the hippocampus of diabetic rats. The administration of resveratrol and probiotics alone or in combination improves antioxidant status and reduces oxidative stress biomarkers in the brain tissue. Moreover, the improvement in the antioxidant capacity is a major contributing factor in the prevention and treatment of neurological disorders [27]. Franco et al. showed that the injection of resveratrol in rats increased their serum TAC levels, compared to the untreated diabetic group [28].

In the present study, an increase was observed in 
the total oxidant level. As a result, an increase was also noted in the activity of SOD, CAT, and GPX enzymes in the hippocampal tissue in untreated diabetic. In a study conducted by Tian et al., treatment with resveratrol increased the activity of SOD and CAT in the hippocampus of diabetic rats, compared to the untreated rats. Moreover, they showed that resveratrol enhanced learning and memory in STZ-induced diabetic rats by improving the activity of oxidative stress enzymes [18]. Similarly, Morshedi et al. showed that the administration of Lactobacillus casei for 12 weeks increased the GPX and SOD serum levels and reduced the level of ROS in diabetic rats [11], which was consistent with the findings of the current study.

The results of this study revealed that lipid peroxidation was higher in untreated diabetic rats, which was in line with the findings of previous studies. In this study, it was revealed that probiotic and resveratrol administration, especially their combined effect, reduced lipid peroxidation levels in the hippocampus of diabetic rats following the increased antioxidant enzyme activity. It is suggested that this combined therapy may be effective in improving the antioxidant system, enhancing MDA metabolism in the brain tissue, and reducing oxidative stress through its synergism effect that may improve memory and learning status in diabetic rats. In this study, the protective effect of probiotics and resveratrol against lipid peroxidation was consistent with the reports provided by previous studies [29].

This study evaluated the therapeutic effects of probiotics and resveratrol on memory and learning performance in diabetic rats. The findings revealed that the combination of probiotics and resveratrol could improve spatial memory and passive avoidance memory, and therefore, may be effective in improving memory and learning in diabetic rats. The results of this study are consistent with those obtained by Wang et al,, which revealed that probiotics influenced memory-related behaviors, including spatial and non-spatial memory [30]. The impairment of memory and learning in diabetic rats is related to hippocampal oxidative stress induced by hyperglycemia [25]. Oxidative damage to the hippocampus of diabetic rats also results in the impairment of cognitive function. Therefore, antioxidants can be effective in preventing neuronal damage and cognitive function [25].

\section{Conclusions}

The present study suggests that these compounds can play important roles in reducing oxidative stress, enhancing antioxidant system activity, decreasing lipid peroxidation, and increasing GLP-1 concentration in hippocampal tissue. Consequently, memory and passive avoidance learning are improved in STZ induced diabetic rats as a result of changes in the activity of hippocampal neurons.

\section{Compliance with ethical guidelines}

All testing process was approved by the Ethics Committee of Hamadan University of Medical Sciences, Hamadan, Iran (IR.UMSHA.REC.1398.117) in accordance with National Institutes of Health Guide.

\section{Acknowledgments}

The authors would like to thank the Hamadan University of Medical Sciences, Hamadan, Iran.

\section{Authors' contributions}

EAO and IK designed the experiment and supervised the experiment. SM participated in animal handling, carried out laboratory tests, and prepared the manuscript. FM performed the pathological tests. IK, AK, and HT managed the analyses of the experiment.

\section{Funding/Support}

This study is a part of an MSc thesis and supported by grants from the Hamadan University of Medical Sciences, Hamadan, Iran (Grant No. 9802241632).

\section{Conflicts of Interest}

The authors declare no conflict of interest.

\section{References}

1. Lee JY, Chan CKY, Chua SS, Ng CJ, Paraidathathu T, Lee KKC, et al. Telemonitoring and team-based management of glycemic control on people with type 2 diabetes: a clusterrandomized controlled trial. Journal of General Internal Medicine. 2020; 35(1):87-94. [DOI:10.1007/s11606-01905316-9] [PMID] [PMCID]

2. Abbasi-Oshaghi E, Khodadadi I, Mirzaei F, Ahmadi M, Tayebinia H, Goodarzi MT. Anethum graveolens L. alleviates sperm damage by limiting oxidative stress and insulin resistance in diabetic rats. The Open Medicinal Chemistry Journal. 2020; 14(1):35-44. [DOI:10.2174/ 1874104502014010035]

3. Davari S, Talaei SA, Alaei H, Salimi M. Probiotics treatment improves diabetes-induced impairment of synaptic activity and cognitive function: behavioral and electrophysiological proofs for microbiome-gut-brain axis. Neuroscience. 2013; 240:28796. [DOI:10.1016/j.neuroscience.2013.02.055] [PMID]

4. Sastre AA, Vernooij RW, Harmand MGC, Marthnez G. Effect of the treatment of type 2 diabetes mellitus on the development of cognitive impairment and dementia. Cochrane Database of Systematic Reviews. 2017 6(6):CD003804. [DOI:10.1002/14651858.CD003804.pub2] [PMID] [PMCID]

5. Ahmed A, Zeng G, Jiang D, Lin H, Azhar M, Faroog AD, et al. Time-dependent impairments in learning and memory in Streptozotocin-induced hyperglycemic rats. Metabolic Brain Disease. 2019; 34(5):1431-46. [DOI:10.1007/s11011-01900448-7] [PMID]

6. Malekiyan R, Abdanipour A, Sohrabi D, Jafari Anarkooli I. Antioxidant and neuroprotective effects of lycopene and insulin in the hippocampus of streptozotocin-induced diabetic rats. Biomedical Reports. 2019; 10(1):47-54. [DOI:10.3892/br.2018.1171] [PMID] [PMCID]

7. Rababa'h AM, Mardini AN, Alzoubi KH, Ababneh MA, Athamneh RY. The effect of cilostazol on hippocampal memory and oxidative stress biomarkers in rat model of diabetes mellitus. Brain Research. 2019; 1715:182-7. [DOI:10.1016/j.brainres.2019.03.025] [PMID]

8. Foster JA, Neufeld KA. Gut-brain axis: how the microbiome influences anxiety and depression. Trends in Neurosciences. 2013; 36(5):305-12. [DOI:10.1016/j.tins.2013.01.005] [PMID] 
9. Hunter K, Hulscher C. Drugs developed to treat diabetes, liraglutide and lixisenatide, cross the blood brain barrier and enhance neurogenesis. BMC Neuroscience. 2012; 13(1):33. [DOI:10.1186/1471-2202-13-33] [PMID] [PMCID]

10. D'Souza A, Fordjour L, Ahmad A, Cai C, Kumar D, Valencia $\mathrm{G}$, et al. Effects of probiotics, prebiotics, and synbiotics on messenger RNA expression of caveolin-1, NOS, and genes regulating oxidative stress in the terminal ileum of formulafed neonatal rats. Pediatric Research. 2010; 67(5):526-31. [DOI:10.1203/PDR.0b013e3181d4ff2b] [PMID]

11. Morshedi M, Saghafi-Asl M, Hosseinifard ES. The potential therapeutic effects of the gut microbiome manipulation by synbiotic containing-Lactobacillus plantarum on neuropsychological performance of diabetic rats. Journal of Translational Medicine. 2020; 18(1):18. [DOI:10.1186/ s12967-019-02169-y] [PMID] [PMCID]

12. Rahmati H, Momenabadi S, Vafaei AA, Bandegi AR, Mazaheri Z, Vakili A. Probiotic supplementation attenuates hippocampus injury and spatial learning and memory impairments in a cerebral hypoperfusion mouse model. Molecular Biology Reports. 2019; 46(5):4985-95. [DOI:10.1007/s11033-019-04949-7] [PMID]

13. Oshaghi EA, Khodadadi I, Tavilani H, Goodarzi MT. Effect of dill tablet (Anethum graveolens L) on antioxidant status and biochemical factors on carbon tetrachloride-induced liver damage on rat. International Journal of Applied and Basic Medical Research. 2016; 6(2):111-4. [DOI: 10.4103/2229-516X.179019] [PMID] [PMCID]

14. Mohammadi A, Bazrafshani MR, Oshaghi EA. Effect of garlic extract on some serum biochemical parameters and expression of npc1l1, abca1, abcg5 and abcg8 genes in the intestine of hypercholesterolemic mice. Indian Journal of Biochemistry \& Biophysics. 2013; 50(6):500-4. [PMID]

15. Abbasi Oshaghi E, Khodadadi I, Saidijam M, Yadegarazari R, Shabab N, Tavilani $H$, et al. Lipid lowering effects of hydroalcoholic extract of anethum graveolens I. and dill tablet in high cholesterol fed hamsters. Cholesterol. 2015; 2015:958560. [DOI:10.1155/2015/958560] [PMID] [PMCID]

16. Goodarzi MT, Khodadadi I, Tavilani H, Abbasi Oshaghi E. The role of Anethum graveolens L. (Dill) in the management of diabetes. Journal of Tropical Medicine. 2016; 2016:1098916. [DOI:10.1155/2016/1098916] [PMID] [PMCID]

17. Abbasi Oshaghi E, Goodarzi MT, Higgins V, Adeli K. Role of resveratrol in the management of insulin resistance and related conditions: Mechanism of action. Critical Reviews in Clinical Laboratory Sciences. 2017; 54(4):267-93. [DOI:10.1080/10408363.2017.1343274] [PMID]

18. Tian X, Liu Y, Ren G, Yin L, Liang X, Geng T, et al. Resveratrol limits diabetes-associated cognitive decline in rats by preventing oxidative stress and inflammation and modulating hippocampal structural synaptic plasticity. Brain Research. 2016; 1650:1-9. [DOI:10.1016/j.brainres.2016.08.032] [PMID]

19. Palsamy P, Subramanian S. Resveratrol, a natural phytoalexin, normalizes hyperglycemia in streptozotocinnicotinamide induced experimental diabetic rats. Biomedicine \& Pharmacotherapy. 2008; 62(9):598-605.
[DOI:10.1016/j.biopha.2008.06.037] [PMID]

20. Mirzaei F, Khazaei M, Komaki A, Amiri I, Jalili C. Virgin coconut oil (VCO) by normalizing NLRP3 inflammasome showed potential neuroprotective effects in Amyloid- $\beta$ induced toxicity and high-fat diet fed rat. Food and Chemical Toxicology. [DOI:10.1016/j.fct.2018.04.064] [PMID]

21. Abbasi-Oshaghi E, Mirzaei F, Mirzaei A. Effects of ZnO nanoparticles on intestinal function and structure in normal/high fat diet-fed rats and Caco-2 cells. Nanomedicine (London, England). 2018; 13(21):2791-816. [DOl:10.2217/nnm-2018-0202] [PMID]

22. Abbasi-Oshaghi E, Mirzaei F, Pourjafar M. NLRP3 inflammasome, oxidative stress, and apoptosis induced in the intestine and liver of rats treated with titanium dioxide nanoparticles: in vivo and in vitro study. International Journal of Nanomedicine. 2019; 14:1919-36. [DOI: 10.2147/IJN.S192382] [PMID] [PMCID]

23. Ravan AP, Bahmani M, Ghasemi Basir HR, Salehi I, Oshaghi EA. Hepatoprotective effects of Vaccinium arctostaphylos against $\mathrm{CCl} 4$-induced acute liver injury in rats. Journal of Basic and Clinical Physiology and Pharmacology. 2017; 28(5):463-71. [DOI:10.1515/jbcpp-2016-0181] [PMID]

24. Shahryari J, Poormorteza M, Noori-Sorkhani A, Divsalar K Abbasi-Oshaghi E. The effect of concomitant ethanol and opium consumption on lipid profiles and atherosclerosis in golden syrian Hamster's aorta. Addiction \& Health. 2013; 5(3-4):83-9. [PMID] [PMCID]

25. Tuzcu M, Baydas G. Effect of melatonin and vitamin E on diabetes-induced learning and memory impairment in rats. European Journal of Pharmacology. 2006; 537(1-3):106-10. [DOI:10.1016/j.ejphar.2006.03.024] [PMID]

26. Isik AT, Soysal P, Yay A, Usarel C. The effects of sitagliptin, a DPP-4 inhibitor, on cognitive functions in elderly diabetic patients with or without Alzheimer's disease. Diabetes Research and Clinical Practice. 2017; 123:192-8. [DOI:10.1016/j.diabres.2016.12.010] [PMID]

27. Gandhi S, Abramov AY. Mechanism of oxidative stress in neurodegeneration. Oxidative Medicine and Cellular Longevity. 2012; 2012:428010. [DOI:10.1155/2012/428010] [PMID] [PMCID]

28. Franco JG, de Moura EG, Koury JC, Trotta PA, Cordeiro A, Souza LL, et al. Resveratrol reduces lipid peroxidation and increases sirtuin 1 expression in adult animals programmed by neonatal protein restriction. The Journal of Endocrinology. 2010; 207(3):319-28. [DOI:10.1677/JOE-100124] [PMID]

29. Mazloom Z, Yousefinejad A, Dabbaghmanesh MH. Effect of probiotics on lipid profile, glycemic control, insulin action, oxidative stress, and inflammatory markers in patients with type 2 diabetes: a clinical trial. Iranian Journal of Medical Sciences. 2013; 38(1):38-43. [PMID] [PMCID]

30. Wang $H$, Lee IS, Braun C, Enck P. Effect of probiotics on central nervous system functions in animals and humans: a systematic review. Journal of Neurogastroenterology and Motility. 2016; 22(4):589-605. [DOI:10.5056/jnm16018] [PMID] [PMCID] 